\title{
Reply to the Letter to the Editor of 0 . Ersen et al. concerning "The effect of patient positioning on the relative position of the aorta to the thoracic spine" by Plataniotis N, et al. (Eur Spine J; 2019: https:// doi.org/10.1007/s00586-018-5812-9)
}

\author{
N. Plataniotis ${ }^{1,2} \cdot$ O. Karargyris ${ }^{1} \cdot$ D. S. Evangelopoulos ${ }^{1} \cdot$ G. Katzouraki $^{1} \cdot$ S. Pneumaticos ${ }^{1}$
}

Received: 2 May 2019 / Accepted: 5 May 2019 / Published online: 28 May 2019

(c) Springer-Verlag GmbH Germany, part of Springer Nature 2019

We have read with great interest the comments of the authors concerning our article titled "The effect of patient positioning on relative position of the aorta to the thoracic spine".

Indeed, radiation is a major concern for spinal surgeons in recent years. New imaging modalities and spinal navigation systems have also been developed in an effort to replace conventional fluoroscopy and reduce radiation doses. The data of our study demonstrated a significant anteromedial migration of the aorta placement when patients are placed in prone positions. We believe that the assessment of the aortic migration provides the surgeon crucial anatomical data and allows to choose optimal operative approach and organize an accurate preoperative plan, thus significantly reducing excessive intraoperative radiation. Study group's safety has always been a priority. The authors explained to all patients the study design, the importance of future findings for proper preoperative planning and further decrease in radiation exposure. Moreover, a low-dose protocol was selected for each scan to minimize radiation exposure. Literature data support that low-dose protocols provide a $60-70 \%$ dose reduction compared to a conventional CT scan. Therefore, all three performed low-dose protocols were almost equal to a standard-dose thoracic spine CT scan. Taking all the above into account, the authors believe that preoperative prone-positioned thoracic CT scans will provide important data for accurate preoperative planning, further reducing intraoperative radiation exposure.

For the performance of this study, the authors chose a modular pudding so that it could be adjusted to patients' physical features. Patients were positioned based on specific anatomical landmarks. Although there was concern that the use of a modular padding could affect the precision of our results, no such finding was observed during statistical analysis of our data.

\section{Compliance with ethical standards}

Conflict of interest The authors declare that they have no conflict of interest.

Publisher's Note Springer Nature remains neutral with regard to jurisdictional claims in published maps and institutional affiliations.

D. S. Evangelopoulos

ds.evangelopoulos@gmail.com

1 3rd Department of Orthopaedic Surgery, University of Athens, KAT Hospital, 2 Nikis str, Kifisia, 14561 Athens, Greece

2 Department of Radiology, KAT Hospital, Athens, Greece 